\title{
Prognostic significance of the combination of preoperative hemoglobin and albumin levels and lymphocyte and platelet counts (HALP) in patients with renal cell carcinoma after nephrectomy
}

Ding Peng ${ }^{1,2+}$ (D) Cui-jian Zhang ${ }^{1,2,3,4+}$, Qi Tang ${ }^{1,2,3,4}$, Lei Zhang ${ }^{1,2,3,4}$, Kai-wei Yang ${ }^{1,2,3,4}$, Xiao-teng Y Y ${ }^{1,2,3,4}$,

Yanqing Gong ${ }^{1,2,3,4}$, Xue-song Li $\mathrm{Li}^{1,2,3,4^{*}}$, Zhi-song He $\mathrm{H}^{1,2,3,4}$ and Li-qun Zhou ${ }^{1,2,3,4^{*}}$

\begin{abstract}
Background: To evaluate the prognostic significance of the novel index combining preoperative hemoglobin and albumin levels and lymphocyte and platelet counts (HALP) in renal cell carcinoma (RCC) patients.

Methods: We enrolled 1360 patients who underwent nephrectomy in our institution from 2001 to 2010. The cutoff values for HALP, neutrophil-to-lymphocyte ratio and platelet-to-lymphocyte ratio were defined by using X-tile software. Survival was analyzed by the Kaplan-Meier method, with differences analyzed by the log-rank test. Multivariate Cox proportional-hazards model was used to evaluate the prognostic significance of HALP for RCC.

Results: Low HALP was significantly associated with worse clinicopathologic features. Kaplan-Meier and log-rank tests revealed that HALP was strongly correlated with cancer specific survival $(P<0.001)$ and Cox multivariate analysis demonstrated that preoperative HALP was independent prognostic factor for cancer specific survival (HR $=1.838,95 \% \mathrm{Cl}: 1.260-2.681, P=0.002$ ). On predicting prognosis by nomogram, the risk model including TNM stage, Fuhrman grade and HALP score was more accurate than only use of TNM staging.

Conclusions: HALP was closely associated with clinicopathologic features and was an independent prognostic factor of cancer-specific survival for RCC patients undergoing nephrectomy. A nomogram based on HALP could accurately predict prognosis of RCC.
\end{abstract}

Keywords: Renal cell carcinoma, Prognosis, HALP, Nephrectomy

\section{Background}

Renal cancer accounts for $2 \%$ to $3 \%$ of all cancers, and the rate of renal cell carcinoma (RCC) has increased by $1.6 \%$ per year for the past 10 years [1]. Approximately $90 \%$ of renal cancer is RCC, and surgery is the only curative treatment. About $20 \%$ of RCC patients have advanced stage disease, and for those with localized RCC, nearly $30 \%$

\footnotetext{
*Correspondence: pineneedle@sina.com; zhoulqmail@sina.com ${ }^{\dagger}$ Equal contributors

'Department of Urology, Peking University First Hospital, No. 8, Xishiku Street, Xicheng District, Beijing 100034, China

Full list of author information is available at the end of the article
}

show recurrence after tumor resection $[2,3]$. Therefore, we need better prognostic models to improve prognosis.

The TNM stage, reflecting tumor invasion, lymph node metastasis and distant metastasis, is the most widely used system for predicting RCC prognosis [2]. However, because of heterogeneous prognoses, the outcomes of some patients with the same stage of cancer may be completely different. Therefore, we need useful biomarkers to increase the prognostic accuracy in RCC.

Increasing evidence supports that inflammation and nutrition are involved in the initiation and progression of various cancers, including RCC [4]. Hematologic parameters including albumin and hemoglobin levels and lymphocytes, 
neutrophils and platelets counts are easily acquired laboratory data reflecting inflammation and nutrition status and have been extensively studied. Numerous studies have reported the prognostic value of serum albumin and hemoglobin levels and lymphocyte and platelet counts for various cancers, including RCC [5-8]. However, the disadvantage of these indicators is that each reflects only one respect of inflammation or nutrition. Further studies found that the combination of those factors in an index such as the prognostic nutritional index (PNI), combining albumin level and lymphocyte count, or the neutrophil-tolymphocyte ratio (NLR), lymphocyte-to-monocyte ratio (LMR) or platelet-to-lymphocyte ratio (PLR) could more accurately predict prognosis than a single index [9-12].

A novel index combining hemoglobin and albumin levels and lymphocyte and platelet counts (HALP) has been found significantly associated with outcomes in colorectal and gastric cancer $[13,14]$. In this study, we investigated the clinical value of this index in RCC patients undergoing nephrectomy.

\section{Methods}

\section{Patients}

We included 1360 patients with histologically confirmed RCC. All patients were underwent nephrectomy in the Department of Urology, Peking University First Hospital, between 2001 and 2010. Clinicopathologic characteristics and laboratory data were collected. Follow-up care including abdominal ultrasonography or abdominal CT, chest X-ray, and laboratory tests was performed at regular intervals (3-month intervals in years 1 to 3,6-month intervals in years 4 to 5 , and 12-month intervals in years 6 to 10 after diagnosis).

\section{Statistical analysis}

Data are presented as number (percentage) for categorical variables and median (interquartile range [IQR]) for continuous variables. HALP was calculated as hemoglobin level $(\mathrm{g} / \mathrm{L}) \times$ albumin level $(\mathrm{g} / \mathrm{L}) \times$ lymphocyte $(/ \mathrm{L}) /$ platelet count (/L), NLR as neutrophil-to-lymphocyte count and PLR as platelet-to-lymphocyte count. The cut-off values for NLR, PLR and HALP were determined by using X-tile v3.6.1 (Yale University) [15]. The X-tile software was able to compare the $P$ values of different cut-off values for a continuous variable and determine the best cut-off value with the most significant $\mathrm{P}$ value. Chi-square test was used to analyze an association of clinicopathologic data with HALP. The Kaplan-Meier survival method was used to estimate cancer-specific survival (CSS), with log-rank test used to test significant differences. The significant variables in the univariate analysis were included in the Cox proportionalhazards regression multivariate survival analyses by Forward LR method. Statistical analyses involved use of SPSS v22.0 (SPSS Inc., Chicago, IL, USA) and $P<0.05$ was considered statistically significant.

\section{Results}

Patient characteristics

We included 1360 patients (952 men, median age 55 years [IQR 46-65]) (Table 1). The median follow-up was 67 months (IQR 36-74) and 139 (10.2\%) patients

Table 1 Basline clinicopathologic characteristics of 1360 patients with renal cell carcinoma (RCC) undergoing nephrectomy

\begin{tabular}{|c|c|}
\hline Characteristics & Total $n=1360$ \\
\hline Age, years, median (IQR) & $55(46-65)$ \\
\hline Female sex & $408(30 \%)$ \\
\hline \multicolumn{2}{|l|}{ Histology subtype } \\
\hline cCRCC & 1228 (90.29\%) \\
\hline non-ccRCC & $132(9.71 \%)$ \\
\hline \multicolumn{2}{|l|}{ Location } \\
\hline left & $626(46.03 \%)$ \\
\hline right & $697(51.25 \%)$ \\
\hline bilateral & $37(2.72 \%)$ \\
\hline \multicolumn{2}{|l|}{ Fuhrman grade } \\
\hline 1 & $374(27.5 \%)$ \\
\hline 2 & 738 (54.26\%) \\
\hline 3 & $237(17.43 \%)$ \\
\hline 4 & $11(0.81 \%)$ \\
\hline \multicolumn{2}{|l|}{ T-stage } \\
\hline 1 & 1015 (74.63\%) \\
\hline 2 & $113(8.32 \%)$ \\
\hline 3 & $225(16.54 \%)$ \\
\hline 4 & $7(0.51 \%)$ \\
\hline \multicolumn{2}{|l|}{ N status } \\
\hline negative & 1327 (97.57\%) \\
\hline positive & $33(2.43 \%)$ \\
\hline \multicolumn{2}{|l|}{ ASA grade } \\
\hline 1 & $192(14.12 \%)$ \\
\hline 2 & $1072(78.82 \%)$ \\
\hline $3 \& 4$ & $96(7.06 \%)$ \\
\hline Sarcomatoid transformation & 65 (4.78\%) \\
\hline Metastasis & $61(4.48 \%)$ \\
\hline Lymphovascular invasion & $100(7.35 \%)$ \\
\hline Necrosis & $419(3.08 \%)$ \\
\hline Hypoalbuminemia & $53(3.9 \%)$ \\
\hline Anemia & $267(19.6 \%)$ \\
\hline NLR, median (IQR) & $2.13(1.60-2.85)$ \\
\hline PLR, median (IQR) & $124.07(97.33-165.22)$ \\
\hline HALP, median (IQR) & $47.48(33.21-63.45)$ \\
\hline
\end{tabular}

Data are $\mathrm{n}(\%)$ unless indicated. $I Q R$ interquartile ratio, $c C R C C$ clear-cell renal cell carcinoma, ASA American Society of Anesthesiologists, NLR neutrophil-to-lymphocyte ratio, PLR platelet-to-lymphocyte ratio, HALP hemoglobin and albumin levels and lymphocyte and platelet counts 
died due to RCC during follow-up. The 5-year estimated CSS was $89.4 \%$ for all patients.

\section{Association of HALP and clinicopathologic features}

We detected cut-off values for HALP, 31.2; NLR, 2.9; and PLR, 198.3 (Fig. 1 and Additional file 1: Figure S1) for dividing patients into low and high HALP, NLR and PLR groups. Decreased HALP level was associated with being female, older age, high Fuhrman grade and high $\mathrm{T}$ stage and $\mathrm{N}$ and $\mathrm{M}$ positive status, sarcomatoid transformation, tumor necrosis, lymphovascular invasion and low NLR or PLR (Table 2).

\section{Association of HALP with patient outcomes}

On univariate analysis, all included clinicopathologic features except for age $(P=0.287)$, gender $(P=0.226)$, histology subtype $(P=0.385)$ and American Society of Anesthesiologists grade $(P=0.964)$ were significantly related to survival outcomes (Table 3). Anemia and hypoalbuminemia, high PLR and low HALP were all significantly associated with worse survival (Fig. 2). On multivariate analyses, prognostic factors for CSS with RCC were Fuhrman grade (HR 1.767, 95\% CI 1.1772.652, $P=0.006)$, T stage $(3.890,2.510-6.030, P<0.001)$, $\mathrm{N}$ stage $(2.480,1.526-4.032, P<0.001)$, M stage $(4.728$, $3.090-7.233, P<0.001)$ and HALP $(1.838,1.260-2.681$, $P=0.002$ ) (Table 3).

\section{Nomogram of HALP-based risk model for RCC}

We next used nomogram to predict 3- and 5-year CSS for individual patients. Independent prognostic factors in the multivariate analysis including Fuhrman grade, TNM status and HALP were included in the nomogram (Fig. 3). Similar to multivariate findings, with nomogram, high Fuhrman grade and advanced TNM status were associated with poor prognosis and high HALP with favorable prognosis.

The calibration curves of the nomogram showed that the predictive probability of 3- and 5-year survival was closely related to the actual 3- and 5-year survival (Fig. 4).
The C-index was 0.881 (95\% CI: $0.853-0.909$ ) by this nomogram compared with $0.846(0.812-0.880)$ with the TNM staging system. Hence, the risk model including TNM stage, Fuhrman grade and HALP had better prognostic prediction accuracy than the only TNM system.

\section{Discussion}

In this study, we evaluated the prognostic significance of the novel index HALP combining hemoglobin and albumin levels and lymphocyte and platelet counts in RCC patients undergoing nephrectomy. HALP was closely associated with clinicopathologic features. Univariate and multivariate analyses demonstrated that HALP was an independent predictor of CSS for RCC patients undergoing nephrectomy. Furthermore, the nomogram based on HALP could predict prognosis more accurately than the TNM system.

There are several known predictive models of RCC such as TNM stage and the Stage, Size, Grading and Necrosis (SSIGN) model [16]. Inflammatory and nutritional indicators based on hematologic parameters such as albumin and hemoglobin levels and lymphocyte, neutrophil and platelet counts were also associated with outcomes with RCC. Moreover, several indicators combined with hematologic parameters, including NLR, LMR, and PLR, were more accurate predictors $[6,9,10,12]$. Recently, indicators combining albumin level with LMR or NLR were found significantly associated with outcomes [17, 18], which suggests better prediction of outcomes by combining inflammatory and nutritional indicators.

Accumulating evidence suggests the important role of the inflammatory response and nutritional status in cancer progression and metastasis. Overall, 30\% of cancer patients were found with cancer-related anemia (CRA) at the time of diagnosis and CRA was associated with more advanced cancer stage [19]. CRA is believed to be associated with chronic blood loss, iron deficiency, and vitamin B12 or folate nutritional deficiency. Meanwhile, imbalanced inflammation regulation, such as increased

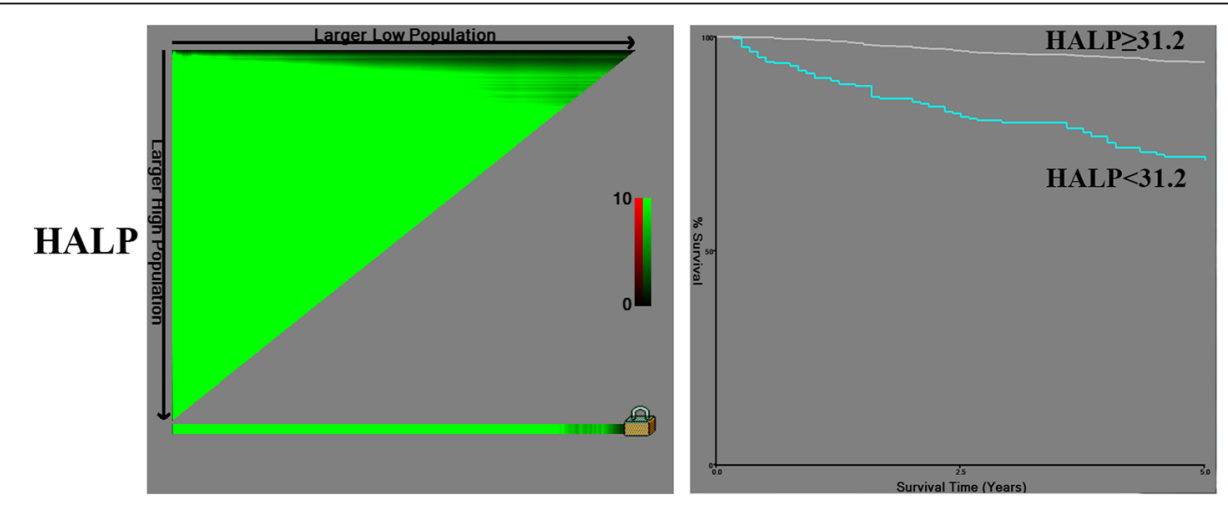

Fig. 1 Cut off value for hemoglobin and albumin levels and lymphocyte and platelet counts (HALP) by using X-tile 
Table 2 Association of baseline clinicopathologic characteristics and HALP

\begin{tabular}{|c|c|c|c|c|}
\hline \multirow[t]{2}{*}{ Variable } & \multirow[t]{2}{*}{$n(\%)$} & \multirow[b]{2}{*}{ low (\%) } & \multicolumn{2}{|l|}{ HALP ${ }^{a}$} \\
\hline & & & high (\%) & $P$ value \\
\hline All patients & 1360 & $291(21.40 \%)$ & 1069 (78.60\%) & \\
\hline Gender & & & & $<0.001$ \\
\hline male & 952 (70.00\%) & $162(17.02 \%)$ & 790 (82.98\%) & \\
\hline female & 408 (30.00\%) & $129(31.62 \%)$ & $279(68.38 \%)$ & \\
\hline Age, years & & & & $<0.001$ \\
\hline$\leq 65$ & 997 (73.31\%) & $186(18.66 \%)$ & 811 (81.34\%) & \\
\hline$>65$ & 363 (26.69\%) & 105 (28.93\%) & 258 (71.07\%) & \\
\hline Histology subtype & & & & 0.695 \\
\hline $\mathrm{cCRCC}$ & 1228 (90.29\%) & $261(21.25 \%)$ & 967 (78.75\%) & \\
\hline non-ccRCC & 132 (9.71\%) & 30 (22.73\%) & 102 (77.27\%) & \\
\hline ASA grade & & & & 0.361 \\
\hline $1+2$ & 1264 (92.94\%) & $268(21.20 \%)$ & 996 (78.80\%) & \\
\hline $3+4$ & $96(7.06 \%)$ & $23(23.96 \%)$ & 68 (76.04\%) & \\
\hline Fuhrman grade & & & & $<0.001$ \\
\hline $1+2$ & 1112 (81.76\%) & 177 (15.92\%) & 935 (84.08\%) & \\
\hline $3+4$ & 248 (18.24\%) & $113(45.56 \%)$ & 130 (54.44\%) & \\
\hline T stage & & & & $<0.001$ \\
\hline $1+2$ & 1128 (82.94\%) & $173(15.34 \%)$ & 955 (84.66\%) & \\
\hline $3+4$ & 232 (17.06\%) & $118(50.86 \%)$ & 113 (49.14\%) & \\
\hline N status & & & & $<0.001$ \\
\hline negative & 1327 (97.57\%) & $270(20.35 \%)$ & 1057 (79.65\%) & \\
\hline positive & $33(2.43 \%)$ & $21(63.64 \%)$ & 12 (36.36\%) & \\
\hline Metastasis & & & & $<0.001$ \\
\hline negative & 1299 (95.51\%) & $258(19.86 \%)$ & 1041 (80.14\%) & \\
\hline positive & 61 (4.49\%) & $33(54.10 \%)$ & 28 (45.90\%) & \\
\hline Sarcomatoid transformation & & & & $<0.001$ \\
\hline absent & $1295(95.22 \%)$ & $245(18.92 \%)$ & $1050(81.08 \%)$ & \\
\hline present & $65(4.78 \%)$ & $46(70.77 \%)$ & 19 (29.23\%) & \\
\hline Tumor necrosis & & & & $<0.001$ \\
\hline absent & $940(69.12 \%)$ & $158(16.81 \%)$ & 782 (83.19\%) & \\
\hline present & $420(30.88 \%)$ & $133(31.67 \%)$ & $286(68.33 \%)$ & \\
\hline Lymphovascular invasion & & & & $<0.001$ \\
\hline absent & $1260(92.65 \%)$ & $249(19.76 \%)$ & 1011 (80.24\%) & \\
\hline present & $100(7.35 \%)$ & $42(42 \%)$ & $58(58 \%)$ & \\
\hline NLR & & & & $<0.001$ \\
\hline high & 317 (23.3\%) & $166(52.37 \%)$ & $151(47.63 \%)$ & \\
\hline low & $1043(76.7 \%)$ & $125(11.98 \%)$ & 918 (88.02\%) & \\
\hline PLR & & & & $<0.001$ \\
\hline high & 195 (14.3\%) & $180(92.31 \%)$ & 15 (7.69\%) & \\
\hline low & 1165 (85.7\%) & 111 (9.53\%) & 1054 (90.47\%) & \\
\hline
\end{tabular}

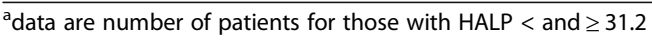


Table 3 Univariate and multivariate analyses of factors associated with cancer-specific survival for RCC patients

\begin{tabular}{lll}
\hline Variable & $\begin{array}{l}\text { Univariate } \\
\text { analysis } P\end{array}$ & Multivariate analysis \\
\cline { 2 - 3 } Age ( $>65$ vs $\leq 65)$ & 0.287 & \\
Gender (female vs male) & 0.226 & \\
Histology subtype (non-ccRCC vs ccRCC) & 0.385 & \\
ASA grade (3 + 4 vs 1+2) & 0.964 & $1.767(1.177-2.652)$ \\
Fuhrman grade (3+4 vs 1+2) & $<0.001$ & $3.890(2.510-6.030)$ \\
T stage (3+4 vs 1+2) & $<0.001$ & $2.480(1.526-4.032)$ \\
N status (positive/negative) & $<0.001$ & $4.728(3.090-7.233)$ \\
M status (positive vs negative) & $<0.001$ & \\
Sarcomatous differentiation (present vs absent) & $<0.001$ & \\
Lymphovascular invasion (present vs absent) & $<0.001$ & \\
Necrosis (present vs absent) & $<0.001$ & \\
Hypoalbuminemia (present vs absent) & $<0.001$ & \\
Anemia (present vs absent) & $<0.001$ & \\
NLR (high vs low) & $<0.001$ & \\
PLR (high vs low) & $<0.001$ & $1.038(1.260-2.681)$ \\
HALP (low vs high) & $<0.001$ &
\end{tabular}
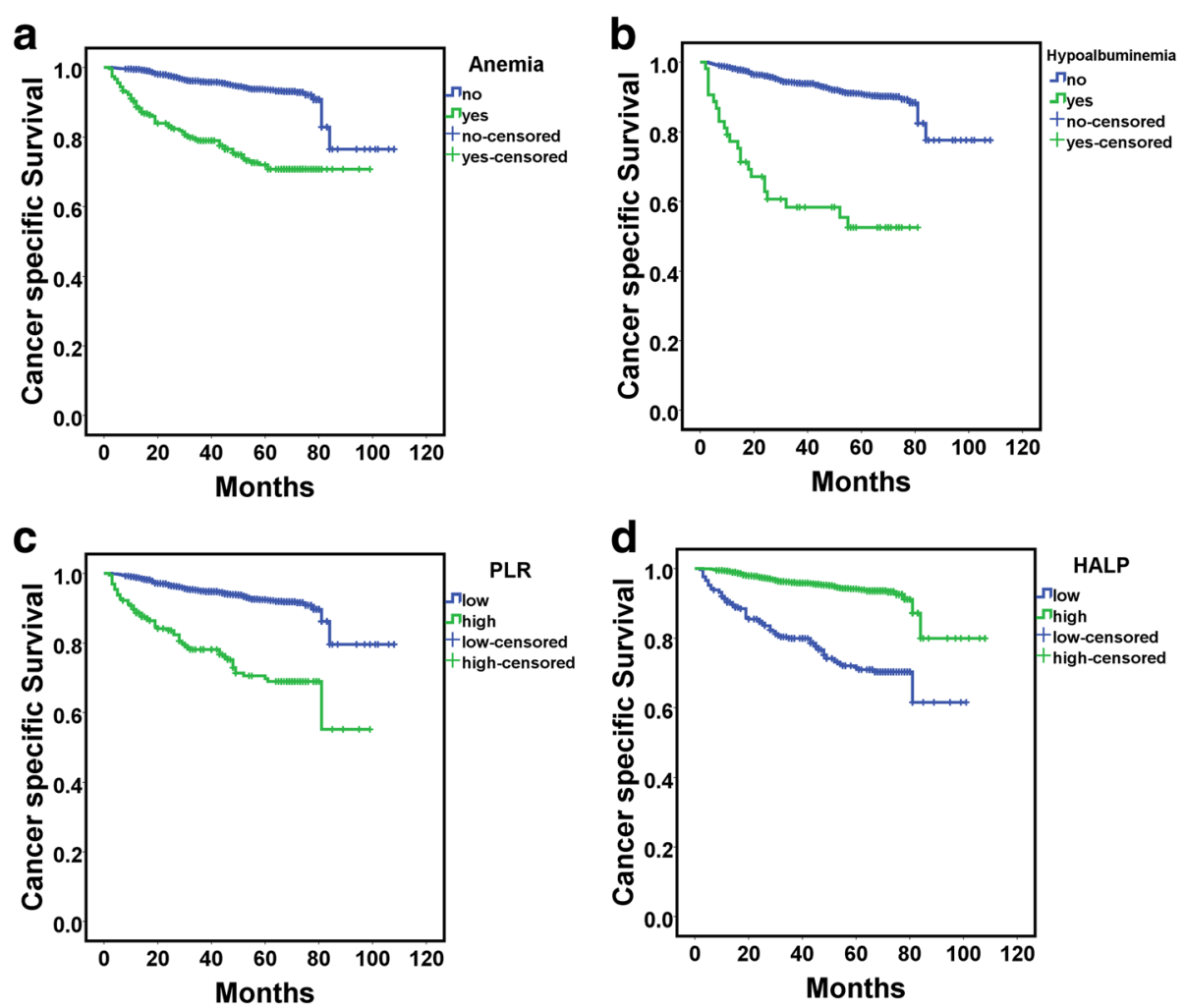

Fig. 2 Kaplan-Meier curves for cancer-specific survival in patients with RCC according to anemia (a), hypoalbuminemia (b), platelet-to-lymphocyte ratio (PLR)(c) and hemoglobin and albumin levels and lymphocyte and platelet counts (HALP) (d) 


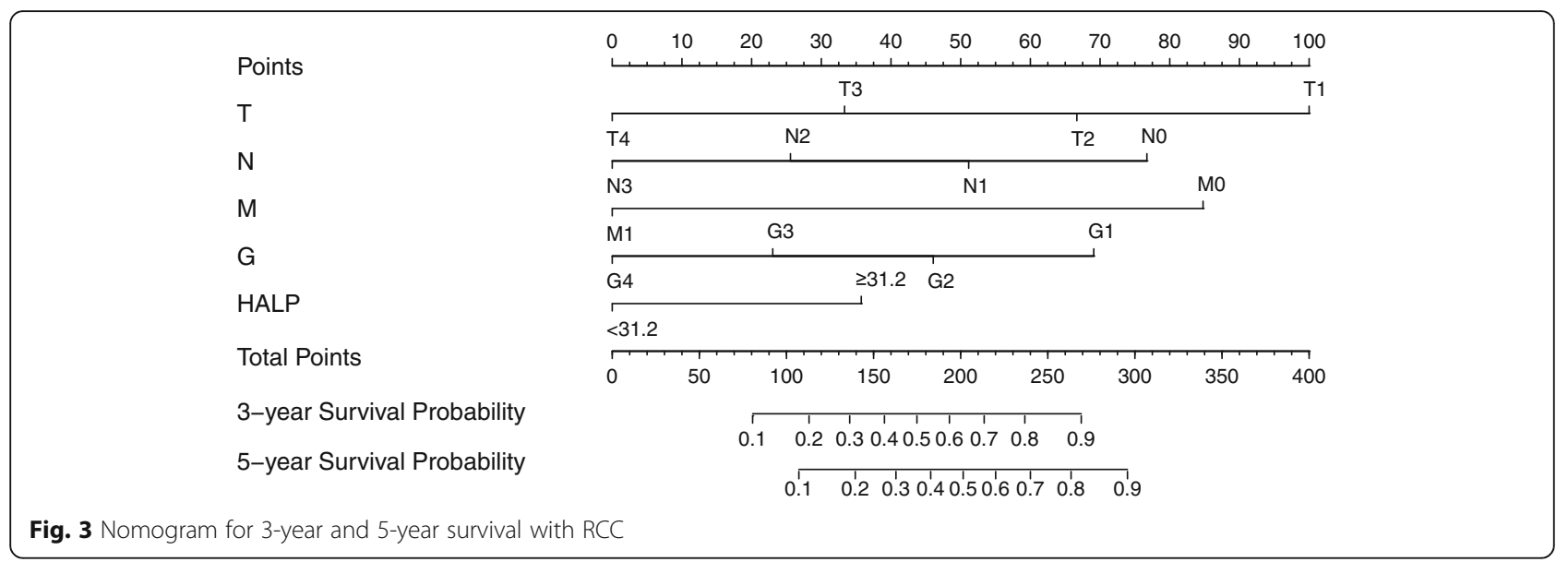

hepcidin and reactive oxygen species stress, in cancer patients is also responsible for CRA [20]. Serum albumin is synthesized specially in the liver and known as a negative acute-phase protein. In addition, systemic factors such as inflammation and stress could affect serum albumin level. Therefore, decreased serum albumin level represents a malnutrition status and also a sustained systemic inflammation response. As important indicators of nutrition and inflammation, anemia and hypoalbuminemia are widely reported to be associated with worse outcomes in various cancers including RCC [7, 8]. Morgan et al. [21] reported that for locoregional RCC patients undergoing nephrectomy, $25 \%$ of patients have anemia and $5.1 \%$ have hypoalbuminemia. The authors also found hypoalbuminemia $(<35 \mathrm{~g} / \mathrm{L})$, unintentional preoperative weight loss $\geq 5 \%$ and preoperative $\mathrm{BMI}<$ $18.5 \mathrm{~kg} / \mathrm{m} 2$ as reflecting nutritional deficiency (ND) and that anemia and ND were independent predictors of overall mortality and disease-specific mortality. Preoperative hypoalbuminemia and anemia were also found to predict transfusion during radical nephrectomy for RCC [5]. We observed 3.9\% hypoalbuminemia and
$19.6 \%$ anemia in our patients, which is consistent with previous study. On univariate analysis, both anemia and hypoalbuminemia were associated with worse survival.

Cancer-related inflammation is considered the seventh hallmark of cancer, playing a conflicting role in tumor initiation and progression in that both tumorantagonizing and -promoting leukocytes can be found [22]. Elevated neutrophil count is associated with cytokine secretion and contributes to tumor angiogenesis, promotion and metastasis. CD4+ and CD8+ T lymphocytes can enhance cancer immune-surveillance to inhibit tumour cell proliferation, invasion and metastasis [23]. Increased neutrophil count and decreased lymphocyte count might be responsible for a weak and insufficient immune response to tumors and strongly associated with a poor survival in advanced cancer [24, 25].

Recent data implied that the activation of platelets is crucial for cancer progression by promoting angiogenesis, extracellular matrix degradation, and release of growth factors, which are essential components of tumor growth and metastatic [26]. In addition, platelets adhering to tumor cells could secrete vascular endothelial growth

\section{a}

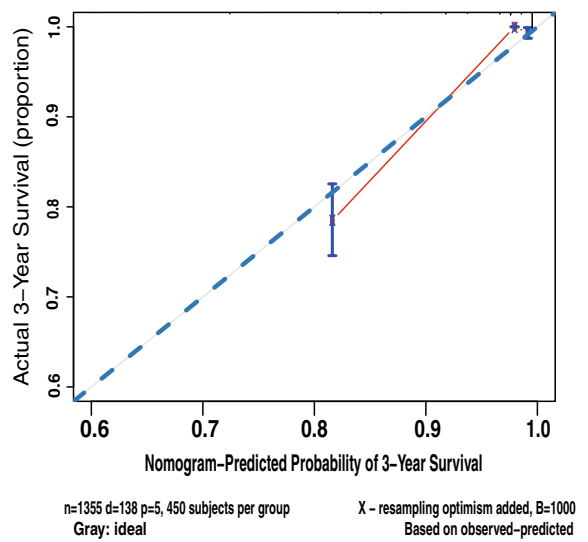

b

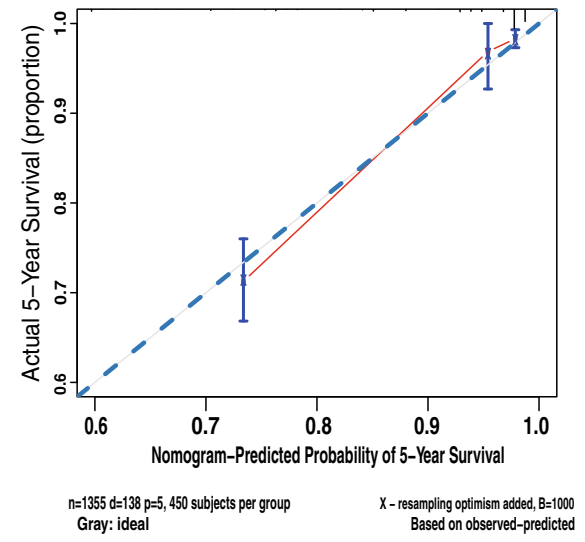

Fig. 4 Calibration curve for 3-year (a) and 5-year (b) survival 
factor(VEGF), which induces microvessel permeability, promotes extravasation of cancer cells, and induces neoangiogenesis [27]. Platelet count was found a significant predictor of RCC-specific mortality [28]. Recently, tumor-educated blood platelets (TEPs) were implicated as central players in the systemic and local responses to tumor growth. As well, the RNA profile in TEPs could provide a valuable platform for liquid biopsy [29].

Systemic inflammation markers including NLR, LMR, and PLR have been found associated with survival in many solid tumors including RCC [6, 10, 30, 31]. Among those indicators, the finding of elevated NLR and PLR indicated increased neutrophil and platelet count and decreased lymphocyte count associated with worse outcomes. In our study, elevated NLR and PLR were associated with worse CSS on univariate analysis. However, on multivariate analyses, HALP rather than NLR and PLR remained an independent prognostic factor.

We used nomogram of independent prognostic factors found on multivariate analyses including HALP and evaluated their accuracy by calibration curves. The predictive accuracy was better with HALP than the TNM system. TNM stage may be an important factor in RCC, but other factors such as HALP could be included and improve the prediction of outcomes.

The major limitations of the present study are its retrospective nature and the single-center design. Additional large and prospective studies are needed to confirm these findings.

\section{Conclusions}

HALP was closely associated with clinicopathologic features of RCC patients undergoing nephrectomy and was an independent prognostic factor of CSS. A nomogram based on HALP could accurately predict prognosis with RCC. Preoperative HALP could be a novel indicator to evaluate the outcome for RCC patients after nephrectomy.

\section{Additional file}

Additional file 1: Figure S1. Cut-off values for neutrophil-tolymphocyte ratio (NLR) and platelet-to-lymphocyte ratio (PLR). (TIFF $6359 \mathrm{~kb})$

\section{Abbreviations}

CRA: cancer-related anemia; CSS: cancer-specific survival; LMR: lymphocyteto-monocyte ratio; NLR: neutrophil-to-lymphocyte ratio; PLR: platelet-tolymphocyte ratio; PNI: prognostic nutritional index; RCC: renal cell carcinoma

\section{Acknowledgements}

We sincerely thank the patients for their participation in this study.

\section{Funding}

This work was supported by the National Natural Science Foundation of China (Grant Number: 81372746 and 81672546).

\section{Availability of data and materials}

The datasets used and/or analysed during the current study available from the corresponding author on reasonable request.

\section{Authors' contributions}

DP: Project development, Data analysis, Manuscript writing. CZ: Project development, Data analysis, Manuscript writing. QT, LZ, KY, XY, YG, ZH: Data collection or management. $X L$ \& $L Z$ : Project development. All authors read and approved the final manuscript.

\section{Ethics approval and consent to participate}

The study was approved by the Ethical Committee of Peking University First Hospital. Written consents to participate in the study from the participants were obtained.

\section{Consent for publication}

We had obtained the consents to publish from the participants to report individual patients' data in any form (including images, videos, voice recordings etc.)

\section{Competing interests}

The authors declare that they have no competing interests.

\section{Publisher's Note}

Springer Nature remains neutral with regard to jurisdictional claims in published maps and institutional affiliations.

\section{Author details}

${ }^{1}$ Department of Urology, Peking University First Hospital, No. 8, Xishiku Street, Xicheng District, Beijing 100034, China. ${ }^{2}$ Institute of Urology, Peking University, Beijing 100034, China. ${ }^{3}$ National Urological Cancer Center, Beijing 100034, China. ${ }^{4}$ Urogenital Diseases (male) Molecular Diagnosis and Treatment Center, Peking University, Beijing 100034, China.

Received: 7 April 2017 Accepted: 8 March 2018

Published online: 15 March 2018

References

1. Motzer RJ, Jonasch E, Agarwal N, Beard C, Bhayani S, Bolger GB, et al. Kidney cancer, version 3.2015. Journal of the National Comprehensive Cancer Network : JNCCN. 2015;13(2):151-9.

2. Ljungberg B, Bensalah $K$, Canfield S, Dabestani S, Hofmann F, Hora M, et al. EAU guidelines on renal cell carcinoma: 2014 update. Eur Urol. 2015;67(5):913-24.

3. Hsieh JJ, Purdue MP, Signoretti S, Swanton C, Albiges L, Schmidinger M, et al. Renal cell carcinoma. Nat Rev Dis Primers. 2017;3:17009-28.

4. Senbabaoglu Y, Gejman RS, Winer AG, Liu M, Van Allen EM, de Velasco G, et al. Tumor immune microenvironment characterization in clear cell renal cell carcinoma identifies prognostic and immunotherapeutically relevant messenger RNA signatures. Genome Biol. 2016;17(1):231-56.

5. Kim K, Seo H, Chin JH, Son HJ, Hwang JH, Kim YK. Preoperative hypoalbuminemia and anemia as predictors of transfusion in radical nephrectomy for renal cell carcinoma: a retrospective study. BMC Anesthesiol. 2015:15:103-10

6. Chang Y, Fu Q, Xu L, Zhou L, Liu Z, Yang Y, et al. Prognostic value of preoperative lymphocyte to monocyte ratio in patients with nonmetastatic clear cell renal cell carcinoma. Tumour Biol. 2016;37(4):4613-20.

7. Corcoran AT, Kaffenberger SD, Clark PE, Walton J, Handorf E, Piotrowski Z, et al. Hypoalbuminaemia is associated with mortality in patients undergoing cytoreductive nephrectomy. BJU Int. 2015:116(3):351-7.

8. Stenman M, Laurell A, Lindskog M. Prognostic significance of serum albumin in patients with metastatic renal cell carcinoma. Med Oncol. 2014; 31(3):841-9.

9. Sun J, Ning H, Sun J, Qu X. Effect of hypertension on preoperative neutrophil-lymphocyte ratio evaluation of prognosis of renal cell carcinoma. Urol Oncol. 2016;34(5):239. e239-215

10. Gunduz S, Mutlu H, Tural D, Yildiz O, Uysal M, Coskun HS. Platelet to lymphocyte ratio as a new prognostic for patients with metastatic renal cell cancer. Asia-Pac J Clin Oncol. 2015;11(4):288-92.

11. Ohno Y, Nakashima J, Ohori M, Gondo T, Hatano T, Tachibana M. Followup of neutrophil-to-lymphocyte ratio and recurrence of clear cell renal cell carcinoma. J Urol. 2012;187(2):411-7. 
12. Hofbauer SL, Pantuck AJ, de Martino M, Lucca I, Haitel A, Shariat SF, et al. The preoperative prognostic nutritional index is an independent predictor of survival in patients with renal cell carcinoma. Urol Oncol. 2015;33(2):68. e61-67

13. Chen XL, Xue L, Wang W, Chen HN, Zhang WH, Liu K, et al. Prognostic significance of the combination of preoperative hemoglobin, albumin, lymphocyte and platelet in patients with gastric carcinoma: a retrospective cohort study. Oncotarget. 2015;6(38):41370-82.

14. Jiang $H$, Li H, Li A, Tang E, Xu D, Chen Y, et al. Preoperative combined hemoglobin, albumin, lymphocyte and platelet levels predict survival in patients with locally advanced colorectal cancer. Oncotarget. 2016;7:72076-83.

15. Camp RL, Dolled-Filhart M, Rimm DL. X-tile: a new bio-informatics tool for biomarker assessment and outcome-based cut-point optimization. Clin Cancer Res. 2004;10(21):7252-9.

16. Frank I, Blute ML, Cheville JC, Lohse CM, Weaver AL, Zincke H. An outcome prediction model for patients with clear cell renal cell carcinoma treated with radical nephrectomy based on tumor stage, size, grade and necrosis: the SSIGN score. J Urol. 2002;168(6):2395-400.

17. Wang $Y Q$, Jin $C$, Zheng HM, Zhou K, Shi BB, Zhang Q, et al. A novel prognostic inflammation score predicts outcomes in patients with ovarian cancer. Clin Chim Acta. 2016;456:163-9.

18. Chang $Y$, An $H$, Xu L, Zhu Y, Yang $Y$, Lin Z, et al. Systemic inflammation score predicts postoperative prognosis of patients with clear-cell renal cell carcinoma. Brit J Cancer. 2015;113(4):626-33.

19. Ludwig H, Van Belle S, Barrett-Lee P, Birgegard G, Bokemeyer C, Gascon P, et al. The European Cancer Anaemia survey (ECAS): a large, multinational, prospective survey defining the prevalence, incidence, and treatment of anaemia in cancer patients. Eur J Cancer. 2004;40(15):2293-306.

20. Maccio A, Madeddu C, Gramignano G, Mulas C, Tanca L, Cherchi MC, et al. The role of inflammation, iron, and nutritional status in cancer-related anemia: results of a large, prospective, observational study. Haematologica. 2015;100(1):124-32.

21. Morgan TM, Tang D, Stratton KL, Barocas DA, Anderson CB, Gregg JR, et al. Preoperative nutritional status is an important predictor of survival in patients undergoing surgery for renal cell carcinoma. Eur Urol. 2011;59(6):923-8.

22. Hanahan D, Weinberg RA. Hallmarks of cancer: the next generation. Cell. 2011;144(5):646-74.

23. Mantovani A, Allavena P, Sica A, Balkwill F. Cancer-related inflammation. Nature. 2008:454(7203):436-44.

24. Hoffmann TK, Dworacki G, Tsukihiro T, Meidenbauer N, Gooding W, Johnson $J T$, et al. Spontaneous apoptosis of circulating T lymphocytes in patients with head and neck cancer and its clinical importance. Clin Cancer Res. 2002;8(8):2553-62

25. Bindea G, Mlecnik B, Fridman WH, Pages F, Galon J. Natural immunity to cancer in humans. Curr Opin Immunol. 2010;22(2):215-22.

26. Labelle M, Begum S, Hynes RO. Platelets guide the formation of early metastatic niches. P Natl Acad Sci USA. 2014;111(30):e3053-61.

27. Suzuki K, Aiura K, Ueda M, Kitajima M. The influence of platelets on the promotion of invasion by tumor cells and inhibition by antiplatelet agents. Pancreas. 2004;29(2):132-40.

28. Karakiewicz PI, Trinh QD, Lam JS, Tostain J, Pantuck AJ, Belldegrun AS, et al. Platelet count and preoperative haemoglobin do not significantly increase the performance of established predictors of renal cell carcinoma-specific mortality. Eur Urology. 2007;52(5):1428-36.

29. Best MG, Sol N, Kooi I, Tannous J, Westerman BA, Rustenburg F, et al. RNASeq of tumor-educated platelets enables blood-based pan-Cancer, multiclass, and molecular pathway Cancer diagnostics. Cancer Cell. 2015; 28(5):666-76.

30. Shin JS, Suh KW, Oh SY. Preoperative neutrophil to lymphocyte ratio predicts survival in patients with T1-2NO colorectal cancer. J Surg Oncol. 2015;112(6):654-7.

31. Hsu JT, Liao CK, Le PH, Chen TH, Lin CJ, Chen JS, et al. Prognostic value of the preoperative neutrophil to lymphocyte ratio in Resectable gastric Cancer. Medicine. 2015:94(39):e1589-95. 